\title{
ORIGINAL ARTICLE \\ Continuous positive airway pressure requirements in patients with tetraplegia and obstructive sleep apnoea
}

\author{
MC Le Guen ${ }^{1,2}$, PA Cistulli ${ }^{3}$ and DJ Berlowitz ${ }^{1}$
}

Study design: Clinic-based retrospective case-control study.

Objectives: To compare continuous positive airway pressure (CPAP) requirements between patients with tetraplegia and able-bodied patients with obstructive sleep apnoea (OSA).

Setting: Melbourne, Australia.

Methods: Diagnostic and CPAP titration polysomnograms of 219 able-bodied, and 25 patients with tetraplegia and OSA were compared for apnoea hypopnoea index (AHI) and CPAP levels required to effectively treat OSA. Demographics and body mass index (BMI) were obtained for each patient. ASIA score and injury date were obtained for patients with tetraplegia.

Results: There was no significant difference in AHI $(P=0.102)$ between the two groups; however, able-bodied patients were significantly older $(P=0.003)$, required significantly higher levels of CPAP to control their OSA $(P<0.001)$ and had higher BMIs $(P=0.009)$ than patients with tetraplegia. In the tetraplegia group, there was no significant correlation between $\mathrm{AHI}$ and effective $\operatorname{CPAP}(r=0.022, P=0.92)$ or between $\mathrm{AHI}$ and $\mathrm{BMI}(r=-0.196, P=0.35)$. There was a significant correlation between effective CPAP and BMI $(r=0.411, P=0.041)$. Among able-bodied patients, over two-thirds $(68.8 \%)$ required $10-16 \mathrm{~cm} \mathrm{H}_{2} 0$ to control their OSA and nearly one-third required over $16 \mathrm{~cm} \mathrm{H} \mathrm{H}_{2} \mathrm{O}$. In contrast, over two-thirds $(68.8 \%)$ in the tetraplegia group required less than $10 \mathrm{~cm} \mathrm{H} \mathrm{H}_{2} \mathrm{O}$ of CPAP to control their OSA.

Conclusion: This retrospective study suggests that OSA patients with tetraplegia require significantly less CPAP to treat their OSA at any given $\mathrm{AHI}$ than those who are able-bodied. This suggests that additional unknown factors may contribute to the high prevalence of OSA in tetraplegia.

Spinal Cord (2012) 50, 832-835; doi:10.1038/sc.2012.57; published online 22 May 2012

Keywords: spinal cord injury; tetraplegia; obstructive sleep apnoea; sleep disordered breathing; continuous positive airway pressure.

\section{INTRODUCTION}

Spinal cord injury (SCI) is a significant cause of morbidity and mortality worldwide. Globally, the annual incidence of SCI ranges from 10 to 40 cases per million population. ${ }^{1}$ In Australia, the annual incidence of SCI is approximately 15 per million with 362 new cases of SCI reported in 2007-2008. Of these, over two thirds were due to trauma and $53 \%$ resulted in tetraplegia. ${ }^{2}$

Obstructive sleep apnoea (OSA) is significantly more common in tetraplegia in comparison with similarly aged able-bodied patients. The reported prevalence of OSA in tetraplegia ranges between 27 and $74 \% .^{3-7}$ Untreated OSA in tetraplegia has significant effects on sleepiness and neurocognitive function, ${ }^{4,5,7-9}$ particularly in areas of attention, concentration, learning skills and memory. ${ }^{10}$ Such neurocognitive deficits may impact on rehabilitation and long-term outcomes. Therefore, it may be important to diagnose and adequately treat OSA among this population.

There is little data regarding continuous positive airway pressure (CPAP) adherence in patients with tetraplegia and OSA. Current data suggest CPAP adherence rates of $23-74 \%$ and those who are more sleepy are more likely to be adherent. ${ }^{9,11}$ Reasons for non-adherence include nasal congestion, claustrophobia, inability to adjust the mask and anxiety. ${ }^{9,11,12}$ There are no data investigating effective CPAP levels required to treat OSA in tetraplegia and whether this differs from able-bodied patients with OSA.

The pathophysiology of OSA in tetraplegia is poorly understood with postulated mechanisms including reduced lung volume, decreased airway patency and male preponderance of SCI. ${ }^{3,7,11} \mathrm{We}$ hypothesised that the mechanisms of OSA in tetraplegia differ from those in able-bodied patients, resulting in a more compliant upper airway and thus lower CPAP requirements. Hence, the aim of this study was to investigate the CPAP levels required to effectively treat OSA in tetraplegia and in able-bodied individuals.

\section{METHODS AND RESULTS}

Clinical data were collected from 219 consecutive, able-bodied patients, and 25 patients with tetraplegia who underwent single-night diagnostic and CPAP titration polysomnography at the sleep unit at the Austin Hospital in 2009. The following parameters were recorded: electroencephalogram (two to four channels), bilateral oculograms, submental and anterior tibial electromyelograms, abdominal and chest respiratory movement (inductive plethysmography), airflow (nasal pressure), pulse oximetry, body position, light readings and sound readings. The studies were sleep staged and respiratory scored by trained sleep scientist employed by Austin Health. Sleep was visually staged in $30 \mathrm{~s}$ epochs, arousals marked and respiratory events scored according to the American Academy of Sleep Medicine criteria. ${ }^{13}$ Overnight CPAP titration

${ }^{1}$ Bowen Centre, Austin Health, Institute for Breathing and Sleep, Melbourne, Victoria, Australia; ${ }^{2}$ Department of Medicine, Dentistry and Health Sciences, The University of Melbourne, Melbourne, Victoria, Australia and ${ }^{3}$ Department of Respiratory and Sleep Medicine, Royal North Shore Hospital, University of Sydney, Sydney, New South Wales, Australia

Correspondence: Dr MC Le Guen, Bowen Centre, Austin Health, Institute for Breathing and Sleep, PO Box 5555, Melbourne, Victoria 3084, Australia. E-mail: mariannick.leguen@austin.org.au

Received 23 December 2011; revised 13 March 2012; accepted 2 April 2012; published online 22 May 2012 
Table 1 Demographics of able-bodied patients and patients with tetraplegia who underwent diagnostic and CPAP titration studies at the Austin in 2009/10

\begin{tabular}{lcccccc}
\hline & \multicolumn{2}{c}{ Able-bodied $(\mathrm{n}=219)$} & & \multicolumn{2}{c}{ Tetraplegia $(\mathrm{n}=25)$} \\
\cline { 1 - 2 } \cline { 5 - 6 } Age (years) & $56.4(13.6)$ & $P=0.11$ & & $46.9(14.2)$ & $P=0.61$ \\
BMI (kg m & -2 & $35.5(8.0)$ & $P<0.005$ & & $30.1(9.3)$ & $P<0.005$ \\
Sex (M/F) & $160 / 59$ & & & $20 / 5$ & \\
AHI (events per h) & $56.9(29.9)$ & $P<0.005$ & & $48.4(23.3)$ & $P=0.13$ \\
CPAP (cm H2O & $14.0(3.5)$ & $P<0.005$ & & $9.0(2.7)$ & $P=0.38$ \\
\hline
\end{tabular}

Abbreviations: $\mathrm{AHI}$ : apnoea hypopnoea index; $\mathrm{BMI}$ : body mass index; CPAP: continuous positive airway pressure.

Values quoted are mean \pm s.d.

polysomnography was used to determine the effective CPAP levels required to abolish respiratory events. The tetraplegia cohort were supine for the entirety of both the diagnostic and CPAP titration studies.

Paired $t$-tests were used to compare apnoea hypopnoea index (AHI), effective CPAP levels, age and body mass index (BMI) between the tetraplegia cohort and able-bodied cohort. The tetraplegia cohort was divided into 'acute' ( $<$ lyr since injury at time of polysomnography) and 'chronic' groups ( $>$ lyr since injury) and compared with the able-bodied cohort ('control'). Analysis of variance (ANOVA) was used to compare BMI and effective CPAP among these three groups. Pearson's correlation was used to test for linear correlations between $\mathrm{AHI}$ and CPAP, AHI and BMI, and CPAP and BMI in the tetraplegia cohort and able-bodied cohort as well as in the 'acute' and 'chronic' tetraplegia groups.

The tetraplegia group included those with acute $(n=15,60 \%)$ and chronic tetraplegia $(n=10,40 \%)$ with complete $(45 \%)$ or incomplete $(55 \%)$ injuries. Table 1 shows the demographics of both groups.

There was no significant difference in AHI $(P=0.102)$ between the two groups. Those from the able-bodied group were significantly older $(P=0.003)$, required significantly higher levels of CPAP to effectively treat their OSA $(P<0.001)$ and had a significantly higher BMI $(P=0.009)$ than the tetraplegia group.

The tetraplegia group were divided into 'acute' ( $<$ lyr since injury) and 'chronic' ( $>$ lyr since injury) and compared with BMI and CPAP. The acute group were significantly lighter (mean $\mathrm{BMI}=29.74 \mathrm{~kg} \mathrm{~m}^{-2}(25.15,34.33)$ ) than the controls (mean $\mathrm{BMI}=35.48 \mathrm{~kg} \mathrm{~m}^{-2}(34.41,36.55)$ ) but not the chronic group (mean BMI $=30.54 \mathrm{~kg} \mathrm{~m}^{-2}(24.29,36.79)$ ). The chronic and control groups did not have a significantly different BMI (ANOVA: $\left.\mathrm{F}_{(2,241)}=4.94, P=0.008\right)$. The control group required significantly higher CPAP (mean $\mathrm{CPAP}=14.0 \mathrm{~cm} \mathrm{H}_{2} \mathrm{O},(95 \%$ confidence interval $13.5,14.5)$ ) to treat their OSA when compared with both the acute (mean CPAP $=9.0 \mathrm{~cm}$ $\mathrm{H}_{2} \mathrm{O}$, (7.7, 10.0)) and chronic (mean $\mathrm{CPAP}=9.0 \mathrm{~cm} \mathrm{H} \mathrm{H}_{2} \mathrm{O}$, (7.0,11.0)) tetraplegia groups. There was no significant difference in CPAP between acute and chronic groups (ANOVA: $\mathrm{F}_{(2,241)}=25.5, P<0.001$ ).

Figure 1 shows the effective CPAP requirements at varying AHI among the tetraplegia and able-bodied groups. In the able-bodied, over two thirds (67.3\%) required $10-16 \mathrm{~cm} \mathrm{H}_{2} \mathrm{O}$ to control their OSA and nearly one quarter required over $16 \mathrm{~cm} \mathrm{H}_{2} 0$ to control their disease. In contrast, less than one third $(31.4 \%)$ of the tetraplegia group required higher than $10 \mathrm{~cm} \mathrm{H}_{2} 0$ of CPAP to control their OSA and none required more than $16 \mathrm{~cm} \mathrm{H}_{2} 0$ of CPAP. In fact, over two thirds (68.8\%) required less than $10 \mathrm{~cm} \mathrm{H}_{2} 0$ of CPAP in order to control their disease.

In the able-bodied group, there was a linear correlation between increasing AHI and the CPAP level required to effectively treat OSA $(r=0.464$, $P<0.001)$. Similarly, there was a linear correlation between increasing AHI and BMI $(r=0.399, P<0.001)$ and between effective CPAP level and BMI $(r=0.387, P<0.01)$. Conversely, in the tetraplegia group there was no significant correlation between AHI and BMI $(r=-0.196, P=0.35)$ or between AHI and effective CPAP level $(r=0.022, P=0.92)$. In addition, there was no significant correlation between AHI and BMI when the tetraplegia group was divided into acute $(r=-0.135, P=0.631)$ and chronic $(r=0.249$,

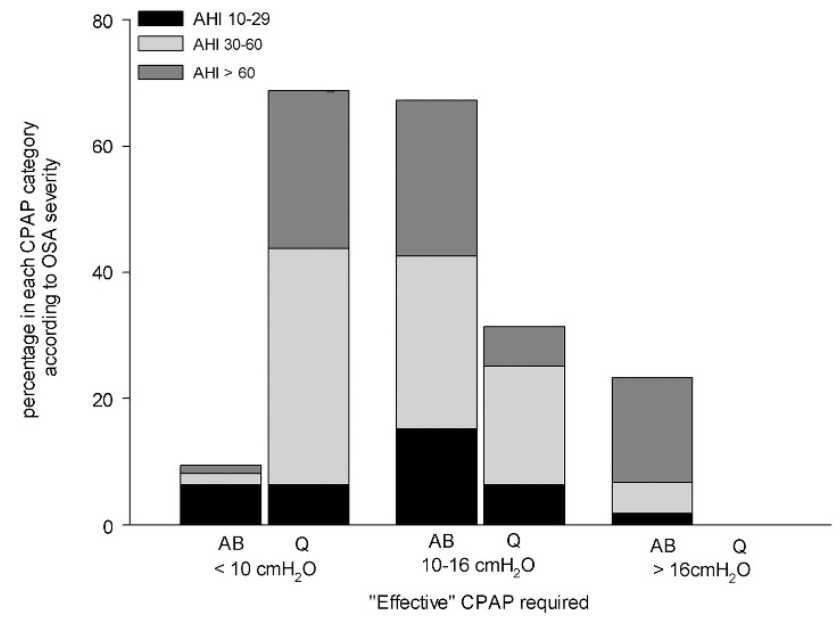

Figure 1 Percentage of patients in different prescribed categories of CPAP level in the able-bodied and quadriplegia groups separated by OSA severity. Note: None of the quadriplegic patients required CPAP pressures $>16 \mathrm{~cm}$ $\mathrm{H}_{2} \mathrm{O}$ to effectively treat their obstructive sleep apnoea. AB, able-bodied; $\mathrm{AHI}$, apnoea-hypopnoea index; CPAP, continuous positive airway pressure; Q, Quadriplegic.

$P=0.49)$. However, there was a significant correlation between effective CPAP level and BMI $(r=0.411, P=0.041)$ in the tetraplegia group as a whole. When only the chronic tetraplegia group is analysed, there is no significant correlation between effective CPAP level and BMI $(r=0.337, P=0.341)$. Figure 2 shows a scatterplot of effective CPAP level vs AHI in both groups and with the tetraplegia group divided into acute and chronic.

\section{DISCUSSION}

This retrospective observational study demonstrates that patients with tetraplegia and OSA require less CPAP to control their OSA than ablebodied patients. There was no significant correlation between AHI and $\mathrm{BMI}$ in the tetraplegia group in contrast to able-bodied patients.

Previous studies have shown a higher prevalence of OSA among patients with tetraplegia, ranging from 27 to $74 \%$. However, only a few studies have also examined CPAP adherence and effective CPAP requirements in this population. ${ }^{5,9,11}$ While the mechanisms of OSA in the able-bodied have been extensively studied, there are no such studies examining the mechanisms underpinning the development of OSA in tetraplegia.

Previous work from our group ${ }^{6}$ has shown that patients with tetraplegia have a high prevalence of OSA, with a recent population survey demonstrating that $74 \%$ of patients with tetraplegia have OSA. This increased to $91 \%$ in those with complete motor and sensory loss, that is, ASIA A injury.

A cross-sectional study of 20 patients with complete tetraplegia by Burns et al. ${ }^{3}$ demonstrated that OSA in tetraplegia is not associated with either BMI or age. However, a subsequent 2001 populationbased, retrospective, case-control study by Burns et al. ${ }^{12}$ of 53 spinal cord injured individuals demonstrated that OSA in tetraplegia is associated with obesity and a higher cervical cord level but not with ASIA impairment. In 2002, Stockhammer et al..$^{5}$ demonstrated that OSA (defined as a respiratory disturbance index $\geqslant 15$ with apnoea index >5) was significantly correlated with age, BMI, neck circumference and time after injury but not with lesion level or ASIA impairment. Our data did not show a significant correlation between AHI and BMI in tetraplegia. Similarly, there was no significant difference in BMI between acute and chronic tetraplegia 

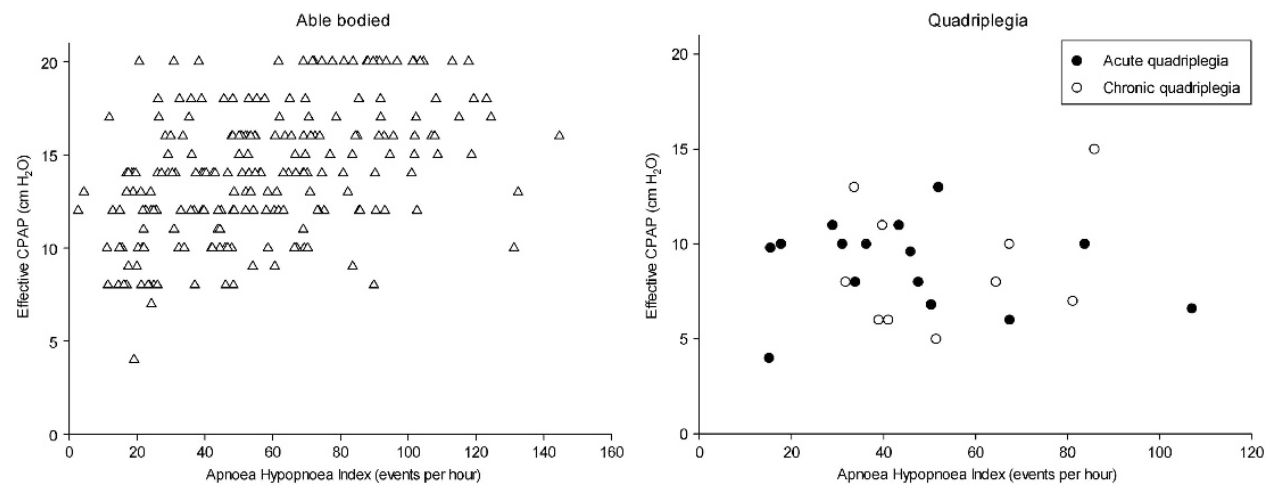

Figure 2 Effective CPAP vs AHI in quadriplegia and abled-bodied with OSA. CPAP, continuous positive airways pressure.

or between chronic tetraplegia and controls. However, there was a significant difference in BMI between acute tetraplegia and controls with acute tetraplegia in the 'overweight' range and controls in the 'severely obese' range. Acute tetraplegia is associated with a hypermetabolic state ${ }^{14,15}$ and a lower body weight. ${ }^{14}$ Weight then gradually increases over time, particularly in the first year after rehabilitation, ${ }^{16}$ such that those with chronic tetraplegia eventually become overweight or obese. ${ }^{17-19}$ Despite the lower BMI in acute tetraplegia, there is still OSA present, necessitating CPAP therapy.

Studies by Burns et al. ${ }^{12}$ and Stockhammer et al. showed poor CPAP acceptance in tetraplegia. Burns et al. reported an adherence rate of $23 \%$, whereas Stockhammer et al. reported an acceptance rate of $35 \%$ (11/34 patients). Those who did not continue therapy cited mask discomfort and anxiety as reasons for discontinuation, whereas Burns et al. has previously hypothesised that impaired hand function may be a contributing factor. ${ }^{3}$ Neither study by Burns et al. nor Stockhammer et al. examined effective CPAP pressures required to treat OSA in tetraplegia.

Berlowitz et al. ${ }^{11}$ examined the feasibility of auto-titrated CPAP for treating OSA in those with acute tetraplegia. Of the 19 participants, 14 had OSA (defined as AHI >10) and of these 14 only $30 \%$ remained adherent with therapy. Those who were adherent were older, had more daytime sleepiness, more severe OSA and a higher BMI. Nasal congestion and claustrophobia were common reasons for non-adherence. Effective CPAP pressures were examined in this study with all but one patient demonstrating a change in CPAP requirements during the 3 -month trial. Our data shows that the tetraplegia group require significantly less CPAP at any given AHI compared with able-bodied individuals with OSA. Furthermore, there is no difference in CPAP requirement between the acute and chronic tetraplegia groups. Although the tetraplegia group demonstrated a correlation between BMI and CPAP requirement this may reflect the higher proportion of acute tetraplegia (associated with lower body weight due to a hypermetabolic state) within this group.

The pathophysiology of OSA in patients with tetraplegia is poorly understood. Suggested mechanisms include reduced lung volume (functional residual capacity), increased neck circumference and abdominal girth, male predominance of SCI and decreased airway patency. ${ }^{3,7,11}$ Data from this study suggests that this population requires less CPAP to effectively treat OSA at any given severity in comparison with able-bodied patients. One possible reason is that individuals with tetraplegia and OSA may have a more compliant upper airway.

Issa et al. ${ }^{20}$ examined the upper airway closing pressure in three groups of snorers: snorers only with nasal obstruction or with alcohol intake, snorers for long periods each night and heavy snorers for long periods each night associated with occasional obstructive apnoeas (mean apnoea index $=4$ per $\mathrm{h}$ ). They demonstrated increasing upper airway collapsibility with increasing severity of snoring. ${ }^{20}$ Schwartz et al. ${ }^{21}$ demonstrated that the pharyngeal critical closing pressure (Pcrit) in normal subjects is negative. Pcrit can be defined as the continuous airway pressure level applied at the nose below which maximal inspiratory airflow ceases and can therefore be considered a measure of airway collapsibility. If Pcrit is negative then negative nasal pressure is required to induce upper airway collapse, and if Pcrit is positive then positive nasal pressure is required to induce upper airway collapse, that is, a lower Pcrit suggests a less collapsible airway. In contrast, Smith et $a .^{22}$ demonstrated a positive Pcrit in patients with OSA, indicating a more collapsible airway during sleep. A study by Gold et al..$^{23}$ examined Pcrit in normal patients and in those with upper airway resistance syndrome (now typically referred to as respiratory effort-related arousals), mild-to-moderate OSA and moderate-to-severe OSA. The mean Pcrit increased as the severity of sleep disordered breathing increased. Therapeutic CPAP levels were also measured in the UARS and OSA groups. Mean therapeutic CPAP requirements increased with increasing severity of sleep disordered breathing with a significant difference between the UARS group and those with moderate-to-severe OSA $(<0.0001) .{ }^{23}$ A study by Sforza et al. ${ }^{24}$ examining the relationship between Pcrit, OSA severity and effective CPAP levels also demonstrated a positive correlation between Pcrit and AHI as well as between effective CPAP levels and Pcrit. Our study demonstrates that patients with OSA and tetraplegia have lower effective CPAP levels, suggesting either that they may also have a lower Pcrit and a less collapsible upper airway compared with able-bodied patients with OSA or that the relationship is altered by unknown factors in tetraplegia.

This study is a non-randomised, observational study and is limited by the small number of individuals $(n=25)$ in the tetraplegia group in comparison to the 219 individuals studied in the able-bodied group. However, this small number is not significantly different from previous studies of OSA in tetraplegia. Furthermore, the tetraplegic group is heterogeneous comprising those with acute and chronic injuries as well as incomplete and complete injuries. Again, previous studies examining OSA in SCI also demonstrate heterogeneity with one study in particular examining patients both with tetraplegia and paraplegia. Finally, while our study raises questions about upper airway collapsibility and the mechanisms of OSA in tetraplegia, it does not directly assess these factors.

\section{CONCLUSION}

This study has demonstrated that patients with tetraplegia require less CPAP to effectively treat their OSA at any given AHI compared to 
able-bodied patients. The mechanisms for OSA in patients with tetraplegia are largely unknown and further studies examining this question would be useful.

\section{DATA ARCHIVING}

There were no data to deposit.

\section{CONFLICT OF INTEREST}

Dr Cistulli is a PI on a multinational clinical trial sponsored by ResMed Inc. and his department has received equipment support for research. Dr David Berlowitz is funded by the Transport Accident Commission (Victoria). Dr MC Le Guen declares no conflict of interest.

1 Wyndaele M, Wyndaele J. Incidence, prevalence and epidemiology of spinal cord injury: what learns a worldwide literature survey? Spinal Cord 2006; 44: 523-529.

2 Norton L. Spinal cord injury, Australia 2007-2008. Injury research and statistics series no. 52. Cat. no. INJCAT 128 AlHW: Canberra, 2010.

3 Burns SP, Little JW, Hussey JD, Lyman P, Lakshminarayanan S. Sleep apnea syndrome in chronic spinal cord injury: associated factors and treatment. Arch Phys Med Rehabil 2000; 81: 1334-1339.

4 Leduc BE, Dagher JH, Mayer P, Bellemare F, Lepage Y. Estimated prevalence of obstructive sleep apnea-hypopnea syndrome after cervical cord injury. Arch Phys Med Rehabil 2007; 88: 333-337.

5 Stockhammer E, Tobon A, Michel F, Esser P, Scheuler W, Bauer W. Characteristics of sleep apnea syndrome in tetraplegic patients. Spinal Cord 2002; 40: 286-294.

6 Berlowitz DJ, Spong J, Gordon I, Howard ME, Brown DJ. Relationships between objective sleep indices and symptoms in a community sample of people with tetraplegia. Arch Phys Med Rehabil 2012 (In press). Accepted 17 February 2012.

7 Short DJ, Stradling JR, Williams SJ. Prevalence of sleep apnoea in patients over 40 years of age with spinal cord lesions. J Neurol Neurosurg Psych 1992; 55 1032-1036.

8 McEvoy RD, Mykytyn I, Sajkov D, Flavell H, Marshall R, Antic R et al. Sleep apnoea in patients with quadriplegia. Thorax 1995; 50: 613-619.
9 Burns SP, Yavari Rad M, Bryant S, Kapur V. Long-term treatment of sleep apnea in persons with spinal cord injury. Am J Phys Med Rehabil 2005; 84: 620-626.

10 Sajkov D, Marshall R, Walker P, Mykytyn I, McEvoy RD, Wale J et al. Sleep apnoea related hypoxia is associated with cognitive disturbances in patients with tetraplegia. Spinal Cord 1998; 36: 231-239.

11 Berlowitz DJ, Spong J, Pierce RJ, Ross J, Barnes M, Brown DJ. The feasibility of using auto-titrating continuous positive airway pressure to treat obstructive sleep apnoea after acute tetraplegia. Spinal Cord 2009; 47: 868-873.

12 Burns SP, Kapur V, Yin KS, Buhrer R. Factors associated with sleep apnea in men with spinal cord injury: a population-based case-control study. Spinal Cord 2001; 39 . 15-22.

13 Sleep-Related Breathing Disorders in Adults: Recommendations for Syndrome Definition and Measurement Techniques in Clinical Research. The Report of an American Academy of Sleep Medicine Task Force. Sleep 1999; 22: 667-689.

14 Rodriguez DJ, Benzel EC, Clevenger FW. The metabolic response to acute spinal cord injury. Spinal Cord 1997; 35: 599-604.

15 Kearns PJ, Thompson JD, Werner PC, Pipp TL, Wilmot CB. Nutritional and metabolic response to spinal cord injury. J Parenter Enteral Nutr 1992; 16: 11-15.

16 Crane DA, Little JW, Burns SP. Weight gain following spinal cord injury: a pilot study. J Spinal Cord Med 2011; 34: 227-232.

17 de Groot S, Post MWM, Postma K, Sluis TA, van der Woude LHV. Prospective analysis of body mass index during and up to 5 years after discharge from inpatient spinal cord injury rehabilitation. J Rehabil Med 2010; 42: 922-928.

18 Gupta N, White KT, Sandford PR. Body mass index in spinal cord injury - a retrospective study. Spinal Cord 2005; 44: 92-94.

19 Groah SL, Nash MS, Ljunberg IH, Libin A, Hamm LF, Ward E. Nutrient intake and body habitus after spinal cord injury: an analysis by sex and level of injury. J Spinal Cord Med 2009; 32: 25-33.

20 Issa FG, Sullivan CE. Upper airway closing pressures in snorers. J Appl Physiol 1984; 57: 528-535.

21 Schwartz AR, Smith PL, Wise RA, Gold AR, Permutt S. Induction of upper airway occlusion in sleeping individuals with subatmospheric nasal pressure. J Appl Physiol 1998; 64: 535-542.

22 Smith PL, Wise RA, Gold AR, Schwartz AR, Permutt S. Upper airway pressure-flow relationships in obstructive sleep apnea. J Appl Physiol 1998; 64: 789-795.

23 Gold AR, Marcus CL, Dipalo F, Gold MS. Upper airway collapsibility during sleep in upper airway resistance syndrome. Chest 2002; 121: 1531-1540.

24 Sforza E, Petiau C, Weiss T, Thibault A, Krieger J. Pharyngeal critical pressure in patients with obstructive sleep apnea syndrome. Am J Respir Crit Care Med 1999; 159: 149-157. 